\title{
Change in Prostaglandin Expression Levels and Synthesizing Activities in Dry Eye Disease
}

\section{Citation}

Shim, Jongwoo, Changhun Park, Hyun Soo Lee, Min Soo Park, Hyung Taek Lim, Sunil Chauhan, Reza Dana, Hyon Lee, and Hyung Keun Lee. 2012. "Change in Prostaglandin Expression Levels and Synthesizing Activities in Dry Eye Disease." Ophthalmology 119 (11) (November): 2211-2219. doi:10.1016/j.ophtha.2012.05.038.

\section{Published Version}

10.1016/j.ophtha.2012.05.038

\section{Permanent link}

http://nrs.harvard.edu/urn-3:HUL.InstRepos:34428276

\section{Terms of Use}

This article was downloaded from Harvard University's DASH repository, and is made available under the terms and conditions applicable to Other Posted Material, as set forth at http:// nrs.harvard.edu/urn-3:HUL.InstRepos:dash.current.terms-of-use\#LAA

\section{Share Your Story}

The Harvard community has made this article openly available.

Please share how this access benefits you. Submit a story.

\section{Accessibility}




\title{
Change in Prostaglandin Expression Levels and Synthesizing Activities in Dry Eye Disease
}

\author{
Jongwoo Shim, MD11, Changhun Park, MS², Hyun Soo Lee, MD³, Min Soo Park, MD², Hyung \\ Taek Lim, MD' ${ }^{1}$, Sunil Chauhan, $\mathrm{PhD}^{3}$, Reza Dana, $\mathrm{MD}^{3}$, Hyon Lee, $\mathrm{MD}^{4}$, and Hyung Keun \\ Lee, MD $^{1,5}$ \\ ${ }^{1}$ Institute of Vision Research, Department of Ophthalmology, Yonsei University College of \\ Medicine, Seoul, Korea. \\ ${ }^{2}$ Clinical Trials Center, Yonsei University College of Medicine, Seoul, Korea. \\ ${ }^{3}$ Schephens Eye Research Institute, Harvard Medical School, Boston, Massachusetts. \\ ${ }^{4}$ Department of Neurology, Seoul National University Hospital, Seoul, Korea. \\ ${ }^{5}$ Institute of Corneal Dystrophy Research, Yonsei University College of Medicine, Seoul, Korea.
}

\begin{abstract}
Objective-To investigate the expression level of prostaglandins (PGs) and their de novo synthesis in dry eye (DE) disease.
\end{abstract}

Design-Cross-sectional case-control study and in vivo mouse experimental study.

Participants-Forty-six eyes from 23 DE patients and 33 eyes from 17 age- and sex-matched controls were studied. Also, DE-induced murine eyes were compared with control eyes.

Methods-Patients completed a symptom questionnaire using a 100-mm visual analog scale (VAS). Nanoliquid chromatography tandem mass spectrometry was used for the quantification of PGE2 and PGD2. A DE disease environmental chamber was used to induce DE in mice. One week after induction, enzyme expressions of cyclooxygenase-1, cyclooxygenase-2 (COX-2), PG E synthase (PGES), and PG D synthase (PGDS) in the lacrimal glands, meibomian glands, and corneas were examined using immunohistochemistry and quantitative real-time polymerase chain reaction (qRT-PCR).

Main Outcome Measures-The mean PGE2 and PGD2 levels in the tears of DE patients were measured and compared with symptom severity scores. Immunohistochemistry staining patterns and qRT-PCR data of DE mice were quantified.

Results-The mean PGE2 level in the tears of DE patients $(2.72 \pm 3.42 \mathrm{ng} / \mathrm{ml})$ was significantly higher than that in the control group $(0.88 \pm 0.83 \mathrm{ng} / \mathrm{ml} ; P=0.003)$. However, the mean PGD2 level in the tears of DE patients $(0.11 \pm 0.22 \mathrm{ng} / \mathrm{ml})$ was significantly lower $(0.91 \pm 3.28 \mathrm{ng} / \mathrm{ml} ; P=$ $0.028)$. The mean PGE2-to-PGD2 ratio correlated strongly with VAS scoring $(P=0.008)$. In DE mice, COX-2 mRNA was significantly higher in ocular surface tissue and lacrimal glands. Furthermore, PGES mRNA was significantly higher in ocular surface tissue, whereas PGDS mRNA was decreased. Immunohistochemistry staining showed elevated COX-2 expression in the

\footnotetext{
(C) 2012 by the American Academy of Ophthalmology

Correspondence: Hyung Keun Lee, MD, Department of Ophthalmology, Kangnam Severance Hospital, 146-92 Dogok-dong, Kangnam-gu, Seoul 135-720, Korea., hyungkeun.lee@gmail.com.

Financial Disclosure(s):

The author(s) have no proprietary or commercial interest in any materials discussed in this article.
} 
lacrimal glands, meibomian glands, corneas, and conjunctivas. Furthermore, PGES expression was found in periductal infiltrated cells of the lacrimal glands and conjunctival epithelium. Also, PGDS expression was decreased in meibomian glands and increased focally in the conjunctival epithelium.

Conclusions-A reciprocal change in PGE2 and PGD2 levels was found in the tears of DE patients, which correlated with patients' symptom scores. These clinical results were supported by increased COX-2 and PGES expression levels found in tear-producing tissues of DE mice.

Financial Disclosure(s)-The author(s) have no proprietary or commercial interest in any materials discussed in this article.

Patients with dry eye (DE) disease typically have symptoms of ocular discomfort ranging from irritation to severe pain. ${ }^{1,2}$ There is an intuitive causal link between the signs of DE observed by clinicians and the severity of symptoms experienced by patients. However, this expectation is challenged by asymptomatic patients with obvious tear film anomalies and extensive ocular surface comprise and, conversely, by patients with intolerable symptoms of dryness in whom only minimal disease can be observed. ${ }^{3}$ Moreover, reliable diagnostic tests to assess symptoms and clinical signs for DE remain controversial. In most clinical practices, DE is evaluated and managed largely on the basis of patients' symptoms. ${ }^{3,4}$

The cornea is highly innervated by sensory nerves that serve important sensory and reflex functions. ${ }^{5}$ Despite the lack of morphologic specialization at the nerve endings, different functional sensory fibers, including polymodal nociceptors, mechanonociceptors, and thermoreceptors, have been identified based on electrophysiologic studies.$^{6-8}$ Generally, activation of thermoreceptors results in a sensation of cooling, whereas activation of mechanoreceptors or polymodal receptors results in ocular surface discomfort and pain. ${ }^{9}$ When noxious stimuli activate sensory afferents in the functional units, a series of coordinated reflexes, including reflex tearing, are triggered to protect the eye from potential damage. ${ }^{10}$ Nociceptors generally are silent electrically and transmit all-or-none action potentials only when stimulated. ${ }^{11}$ To activate nociceptors, proper and adequate stimuli are required. Heat or cold, intense pressure or squeezing, and irritating chemicals can result in depolarizing nociceptor terminals. ${ }^{11,12}$ The known chemical nociceptor activators are capsaicin, bradykinin, histamine, and prostaglandin (PG). ${ }^{11-16}$

Prostaglandins are short-lived lipid mediators that exert a multitude of biological functions. They are synthesized from arachidonic acid after its mobilization from the cell membrane after a host of stimuli, including inflammatory stimuli. ${ }^{17}$ Use of PG analogs (e.g., latanoprost) in ocular disease showed that these drugs cause ocular inflammation and various side effects in ocular tissues. ${ }^{18,19}$ Common side effects of PG analogs are ocular pain, discomfort, and conjunctival injection, which are the prevalent symptoms of DE. ${ }^{20}$ Increased levels of proinflammatory cytokines, such as interleukin (IL)-1, IL-6, and tumor necrosis factor a have been detected in the tear fluid and conjunctival epithelium of DE patients. ${ }^{4,21}$ Interestingly, for the induction of PGs, inflammatory stimuli such as IL-1 are known to induce cyclooxygenase (COX) and PG rapidly. ${ }^{22}$ Moreover, a previous population-based study reported that subjects who took oral aspirin for managing atherosclerosis and hypertension reported low DE symptoms and signs. ${ }^{23}$ Despite the inflammatory nature of DE, the level of PGs and the exact role of PGs in DE have not been determined.

This study investigates PG levels, including PGE2, PGD2, and PGI2, in DE patients and determines whether different levels of PGs are related to subjective symptom scores. In addition, the level of PG synthesizing enzymes in ocular surfaces and lacrimal glands was investigated using an induced mouse environmental chamber model of DE. 


\section{Patients and Methods}

\section{Patients and Examinations}

The study was conducted at 2 independent sites. The cross-sectional, case-control clinical trial was conducted at the Severance Hospital, Department of Ophthalmology, Yonsei University College of Medicine, Seoul, Korea. The in vivo animal DE work was performed at Schepens Eye Research Institute, Harvard Medical School, Boston, Massachusetts. All procedures conformed to the tenets of the Declaration of Helsinki, and informed consent was obtained from all patients after gaining approval from the institutional review board. A total of 23 non-Sjögren-type DE patients completed the study ( 9 men, 14 women), with a mean age \pm standard deviation of $57.0 \pm 12.9$ years (range, $35-84$ years). Inclusion criteria were: 1 or more DE-related symptoms, including tightness, foreign body sensation, irritation, red eye, itching sensation, blurring, or pain; Schirmer test results (with anesthesia) of $8 \mathrm{~mm}$ in 5 minutes or less in both eyes; tear film break-up time of 5 seconds or less in both eyes; and typical keratoconjunctivitis sicca pattern of superficial punctuate erosion of conjunctiva or cornea. Patients were excluded for history of using any eye drops in the most recent month; infection or trauma; ocular or other surgery within the previous 6 months; severe blepharitis with meibomian gland dysfunction; blinking abnormality (e.g., Parkinson's disease, facial nerve palsy); severe pterygium or uncontrolled systemic disease; or if they were pregnant or lactating. The control group comprised 33 eyes from 17 age- and sex-matched healthy subjects ( 6 men, 11 women) who had no history of ocular or systemic diseases, no subjective symptoms of ocular surface dryness, a tear film break-up time of more than 8 seconds, and a Schirmer value of more than $10 \mathrm{~mm}$ per 5 minutes. The mean age of control subjects was 53.0 \pm 17.0 years (range, 26-73 years). The demographic data for included patients are in Table 1 (available at http://aaojournal.org).

For baseline evaluation, tear film break-up time, Schirmer test, fluorescein staining using biomicroscopy, tear sampling, and intraocular pressure were measured in both eyes of DE patients and of healthy normal controls. Symptom severity scores also were obtained using the visual analog scale (VAS) from DE patients who were instructed to grade their symptoms on a $10-\mathrm{cm}$ bar scale $(0 \mathrm{~cm}$, no symptoms; $10 \mathrm{~cm}$, severe and intolerable pain) and to evaluate right and left eyes separately. To determine the ocular pain susceptibility and threshold between healthy controls and DE patients, standard $\mathrm{pH}$ solutions ( $\mathrm{pH}$ 4.5-7.0) were prepared at 0.5 -scale intervals with a phosphate buffer-based formula (Samil-Allergan, Inc., Seoul, Korea), and 1 drop of each $\mathrm{pH}$ solution from $\mathrm{pH} 7.0$ to the lower $\mathrm{pH}$ values were administered into the eye. The threshold of ocular irritation at the $\mathrm{pH}$ when patients first reported irritation and the suprathreshold at 0.5 units lower than the threshold were measured.

During the test period, all evaluations were performed in a masked fashion. The DE classification and tear sampling were performed by one author (C.P.). Another author (J.S.), who was blinded to healthy control or DE status, evaluated threshold and pain scores at the suprathreshold of the patients. Blinded data were analyzed by another author (H.K.L.).

\section{Tear Sampling and Measurement of Prostaglandin E2, Prostaglandin D2, and Prostaglandin I2}

To measure the concentration of PGs E2, D2, and I2 in tears, a bonDE 2.0 $\times 10$-mm polyester fiber rod (Transorb Wicks; Filtrona, Richmond, VA) was used to collect tear fluid as reported previously. ${ }^{24} \mathrm{~A}$ polyester wick was introduced into the lower conjunctival sac and was removed and placed in a $1.5-\mathrm{ml}$ Eppendorf tube, which was stored at $-70^{\circ} \mathrm{C}$ until mass spectrophotometric assay. All nano-liquid chromatography tandem mass spectrometry experiments were carried out on an Applied Biosystem/MDS Sciex 4000 Qtrap quadrupole 
mass spectrometer (AB/MDS Sciex, Concord, Canada) equipped with a turboion spray source. The mass spectrometer was coupled to an Agilent 1200 series high-performance liquid chromatography system (Agilent Technologies, Santa Clara, CA). Data were acquired and processed using Analyst software (version 1.4.2; AB/MDS Sciex). Chromatographic separation was achieved with a Synergi Hydro-RP (C18) 50×2.1-mm inner diameter, 4- $\mu \mathrm{m}$ $80-\AA$ (angstrom units) particles column (Phenomenex, Torrance, CA) at $40^{\circ} \mathrm{C}$. The analysis time was 7 minutes and the working mass spectrometer mode was multiple reaction monitoring. The mobile phase was a binary gradient using $40 \%$ acetonitrile as solution A and $100 \%$ methanol as solution B at a 60:40 (volume/volume) ratio. The flow rate was 0.6 $\mathrm{ml} / \mathrm{minute}$.

\section{Induction of Dry Eye and Securing Eye and Ocular Adnexal Tissues Using a Mouse Dry Eye Chamber Model}

Female (C57BL/6) mice 6 to 8 weeks of age (Charles River Laboratory, Wilmington MA) were used in accordance with the standards in the Association for Research in Vision and Ophthalmology Statement for the Use of Animals in Ophthalmic and Vision Research. The research protocol was approved by the Schepens Eye Research Institute Animal Care and Use Committee. As described previously, DE was induced in the mice by placing them in a controlled environment chamber. ${ }^{25}$ To achieve maximum ocular surface dryness, in addition to the controlled environment chamber, the mice were given subcutaneous injections of 0.1 $\mathrm{ml}$ scopolamine hydrobromide, $5 \mathrm{mg} / \mathrm{ml}$ (Sigma-Adlrich Chemical Co., St. Louis, MO), 3 times daily for the duration of the experiment.

During the DE induction, the mice were killed at 3 days and 7 days after induction. The lids, eyeballs, and lacrimal glands were collected. Each tissue was halved, with half fixed with $3.7 \%$ paraformaldehyde and stored until immunostaining. The other half was stored at $-70^{\circ} \mathrm{C}$ for quantitative real-time polymerase chain reaction analysis. To investigate the effect of COX-2 inhibitor, 3 different concentrations of celecoxib (Sigma-Aldrich, Inc.) solution $(0.1 \mu \mathrm{g} / \mathrm{ml}, 1.0 \mu \mathrm{g} / \mathrm{ml}$, and $10.0 \mu \mathrm{g} / \mathrm{ml})$ were administered into 6 eyes of 3 mice 3 times daily for 1 week in 2-week DE-induced mice.

\section{Immunohistochemical Staining for Prostaglandins}

Lids, conjunctivas, corneas, and lacrimal glands were harvested and analyzed by immunohistochemical staining. Four to 6 eyeballs with lids and lacrimal glands from 4 to 6 mice were used for each antibody of each group (control and DE-induced group). Histologic sections (5 to $7 \mu \mathrm{m}$ ) were collected on poly-L-lysine-coated slides and deparaffinized, and sections were rehydrated with a xylene-grade alcohol scale and were rinsed with phosphatebuffered saline. Intrinsic peroxidase activity was blocked with $3 \% \mathrm{H}_{2} \mathrm{O}_{2}$ for 5 minutes at room temperature, and the sections were exposed to primary antibodies: rat monoclonal antimouse COX-1 (Cayman, Ann Arbor, MI), COX-2 (Abcam, Cambridge, UK), PGE synthase (PGES; Cayman, Ann Arbor, MI), or PGD synthase (PGDS; Cayman, Ann Arbor, MI). All antibodies were diluted 1:300 and were incubated overnight. After washing in $0.05 \%$ Tween 20, sections were exposed to universal biotin-conjugated antibodies for 30 minutes at room temperature. Sections were incubated with peroxidase-conjugated streptavidin for 20 minutes at room temperature. Color development was carried out with diaminobenzidine chromogen and $0.05 \% \mathrm{H}_{2} \mathrm{O}_{2}$. Sections were counterstained with Meyer's hematoxylin (DAKO, Glostrup, Denmark). Human skin and colon tissue were used as positive controls. Light microscope (Axio Imager 2, Carl Zeiss, Oberkochen, Germany) was used for examination. 


\section{Tissue RNA Extraction and Quantitative Real-Time Polymerase Chain Reaction}

Four to 6 corneas and lacrimal glands from 4 to 6 mice were involved in each group (control, 3-day DE induction, 7-day DE induction, and varying celecoxib concentration groups). Each experiment was repeated 3 times. RNA was isolated with RNeasy Micro Kit (Qiagen, Hilden, Germany) from mouse corneas and lacrimal glands and reverse transcribed using a Superscript III Kit (Invitrogen, Carlsbad, CA). Quantitative real-time polymerase chain reaction was performed using Taq-Man Universal PCR Mastermix and preformulated primers for COX-1 (Mm04225243_g1; Applied Biosystems, Foster City, CA), COX-2 (Mm03294838_g1), PGE2 synthase (Mm00452105_m1), PGD2 synthase (Mm01330613_m1), and GAPDH (Mm99999915_g1).

\section{Statistical Analysis}

Basic characteristics of the study population, including healthy controls and DE patients, were reported for each variable by descriptive statistics using the Fisher exact test for frequency data, the $t$ test for continuous data, and the Mann-Whitney $U$ test for nonnormally distributed data. Correlations of ocular irritation scores and concentration of PGE2-to-PGD2 were analyzed by linear regression analysis with a $95 \%$ confidence interval. The 1-way analysis of variance was used to assess mRNA expression. All statistical tests were 2-sided at the $95 \%$ confidence interval and were performed using the SAS software version 9.13 (SAS Inc, Cary, NC).

\section{Results}

Prostaglandin E2, Prostaglandin D2, and Prostaglandin I2 Concentration in Dry Eye Patient Tears

In DE patients, PGE2 concentration demonstrated a 3.1-fold increase compared with normal controls (mean \pm standard deviation, $2.72 \pm 3.42 \mathrm{ng} / \mathrm{ml}$ vs. $0.88 \pm 0.83 \mathrm{ng} / \mathrm{ml} ; P=0.003$; Fig 1A). However, PGD2 was significantly decreased in DE patients $(0.11 \pm 0.22 \mathrm{ng} / \mathrm{ml})$ compared with normal controls $(0.91 \pm 3.28 \mathrm{ng} / \mathrm{ml} ; P=0.028$; Fig 1B). In both normal controls and DE patients, PGI2 was not detected. Some representative liquid chromatography tandem mass spectrometry profiles are shown in Figure 2 (available at http://aaojournal.org).

\section{Correlation of Patients' Ocular Irritation Symptoms and Prostaglandin Levels}

Prostaglandin E2 is an inflammatory autacoid that elicits pain or irritative symptoms by lowering the nerve ending firing threshold. ${ }^{26}$ Therefore, whether patients' symptom scores correlated with tear PG levels was determined. Although PGE2 or PGD2 concentrations alone did not correlate with symptom score values (Table 2), the ratio of PGE2-to-PGD2 levels correlated significantly with the VAS score. The DE patients with a higher VAS score tended to have high PGE2-to-PGD2 ratios in their tears.

To determine the effect of PGE2-to-PGD2 levels on nerve terminal firing by an irritation stimulus, an ocular stimulation challenge test was carried out using standard $\mathrm{pH}$ solutions. Because a commercial antiglaucoma medication $\mathrm{pH}$ is 5.6, the standard solution was prepared from 5.0 to 8.0 , with a 0.5 -unit scale. From the challenge test, DE and normal controls felt ocular irritation at similar $\mathrm{pH}$ values (pH 5.59 \pm 0.49 and $\mathrm{pH} 5.68 \pm 0.40$, respectively; $P=0.63$ ) with the same intensity (VAS score, $1.81 \pm 0.98$ vs. $1.73 \pm 1.19 ; P=$ 0.84; Fig 3, available at http://aaojournal.org). However, the intensity of irritation was much higher for DE patients $(2.41 \pm 1.46)$ than normal patients $(1.31 \pm 0.60)$ at suprathreshold levels (challenged by $0.5 \mathrm{pH}$ units lower than the threshold; $P=0.012$; Fig 3 , available at http://aaojournal.org). 
mRNA of Cyclooxygenase-1, Cyclooxygenase-2, Prostaglandin E2 Synthase, and Prostaglandin D2 Synthase in Dry Eye Mice

Dry eye patients had higher PGE2-to-PGD2 levels in their tears. Because the study of human tissue was not feasible, PG enzyme production in the ocular surface and lacrimal glands of a DE mouse model was investigated. In these tissues, COX-2 and PGE mRNA levels were elevated significantly in DE mice compared with normal mice $(P<0.001$; Fig 4$)$. At 3 days after DE stimulus, COX-2 activity was higher than at 7 days (Fig 4). The PGE mRNA levels in the cornea also were elevated by a DE environment. In contrast to COX-2 and PGES, PGDS mRNA was decreased significantly at 3 and 7 days in the DE condition (Fig 4). However, COX-1 mRNA did not show any difference (data not shown).

\section{Immunohistochemical Staining for Cyclooxygenase-1, Cyclooxygenase-2, Prostaglandin E2 Synthase, and Prostaglandin D2 Synthase in Dry Eye Mice}

To define the pattern of PG production in different ocular tissues, immunohistochemical staining for PG enzyme induction was performed, and the results are summarized in Table 3. In DE mice, COX-2 staining was increased in the lacrimal gland, meibomian gland, and corneal and conjunctival epithelium (Fig 5). Furthermore, COX-2 stained mainly acinar cells of the glandular tissue and ductal epithelium of lacrimal glands (Fig 5B) and meibomian glands (Fig 5D). In the normal corneal epithelium, COX-2 stained only basal epithelium of the limbal area (Fig 5E). However, in the DE condition, COX-2-positive cells were found throughout the entire epithelial layer (Fig 5F, black arrow) and some anterior stromal keratocytes (Fig 5F, asterisk). However, although some parts of the conjunctival epithelium showed COX-2 expression (Fig 5H, arrowhead), the COX-2 expression in the conjunctiva was seen faintly in some epithelial cells, with few stromal staining cells showing reduced staining intensity compared with that of the cornea.

Interestingly, PGES were not found in acinar cells of lacrimal glands in DE mice (Fig 6, available at http://aaojournal.org). However, infiltrated cells near the secretary duct and ductal epithelium showed PGES-positive cells (Fig 6B). Also, PGES was found in some conjunctival cells in DE mice (Fig 6D), but not in the corneas and meibomian glands (Fig $6 \mathrm{~F})$.

The PGDS-positive cells were found in meibomian gland in both normal and DE conditions (Fig 6G, H), but not in lacrimal gland acinar or ductal cells. In the ocular surface, some conjunctival epithelial cells showed PGDS expression (Fig 6J, black arrowhead), especially in the limbal area. However, few corneal epithelial cells were found to have PGDS in DE mice (Fig 6L).

\section{Effect of Topical Celecoxib on Prostaglandin E2 and Prostaglandin D2 Synthase mRNA Levels in the Lacrimal Gland}

Finally, COX-2 activity was determined by treatment with a topical COX-2 inhibitor, celecoxib. After 2 weeks of inducing DE, 3 different concentrations of celecoxib were applied topically to DE-induced mice eyes for another week. The COX-2 activity in the cornea was decreased significantly by topical celecoxib at a relatively low concentration $(0.1$ $\mu \mathrm{g} / \mathrm{ml} ; P=0.022 ;$ Fig $7 \mathrm{~A}$ ). However, in lacrimal glands, COX-2 activity was not inhibited as effectively by celecoxib as in the cornea. A higher concentration $(10 \mu \mathrm{g} / \mathrm{ml})$ of celecoxib decreased the COX-2 activity in the lacrimal gland $(P=0.048$; Fig $7 \mathrm{~B})$.

\section{Discussion}

Because PGs are known to be involved with nociceptor excitation and have not been measured in the ocular surface in DE, the principal goal of this study was to determine PG 
levels accurately and to assess the correlation between ocular irritation symptoms and PG concentrations. Elevated PGE2 and downregulated PGD2 were found in tears from DE patients. The values correlated with the patients' irritation scores. In addition, using an in vivo DE mouse model, the synthesizing enzymes for PGE2 were upregulated in the lacrimal glands and ocular surfaces. However, COX-1 expression was not different between normal and DE mice by either mRNA measurement or immunohistochemical staining, for both corneas and lacrimal glands (data not shown). Because of the short half-life of PGs, mass spectrometry coupled with an nano-liquid chromatography tandem mass spectrometry and a high-performance liquid chromatography system were used. To the authors' knowledge, this system is currently the most accurate method for measuring small molecules with extremely low concentrations, such as PGs. On the ocular surface, PGs were in relatively higher concentrations, even in disease-free subjects.

Several studies have investigated the role of PGs in ocular surface diseases, including DE. However, most focused on the anti-inflammatory role of the polyunsaturated fatty acid phospholipase A, and PGE1. ${ }^{27-29}$ In contrast to PGE1, which has anti-inflammatory properties, PGE2 is a PG with a significant role in inflammation. ${ }^{26,30}$ Indeed, PGE2 is a key mediator of pain and pyresis resulting from inflammation. Therefore, inhibitors of PGE2 synthesis such as nonsteroidal anti-inflammatory drugs give relief from fever and pain. ${ }^{30}$ Although the pathophysiologic role of PGE2 in DE could not be demonstrated clearly, considering the primary function of PGE2 in inflammation, the elevated PGE2 levels in DE may be involved in the maintenance of nociceptor excitation and the inflammatory pain state. The ocular surface is damaged easily by mild desiccation, inflammation, and microbes. Damaged cells may induce PGE2 at the injured region by releasing chemical mediators or by direct physical stimulus. ${ }^{3}$ Furthermore, PGE2 concentration at the ocular surface may be elevated more quickly than in other tissues because of tear film evaporation and the narrow conjunctival sac, which may prolong and worsen inflammation. Therefore, although this hypothesis needs further investigation, compared with other body sites, even a small amount of PGE2 is likely to be sufficient to elicit and maintain the inflammatory pain state.

In addition, the PGE2-to-PGD2 ratio correlated better with the subjective irritation score than the PGE2 or PGD2 concentration. Functionally, these prostanoids have opposite effects in the inflammatory process. ${ }^{31,32}$ For instance, PGE2 and its synthesizing enzyme PGES are involved in the generation of inflammation. However, PGDS is a protective factor in reducing inflammatory disease, such as atherosclerosis. ${ }^{31}$ Because the synthesizing enzyme of each prostanoid is an isomerase in the PG synthesizing pathway and the next step of COX-2 activity in the PG synthesis pathway, the expression pattern of the prostanoids is reciprocal. ${ }^{31}$ The elevation of one PG prevents elevation of the other, which results in amplification of the specific effect of the elevated PG. Of note, proinflammatory stimuli (e.g., tumor necrosis factor a) found within inflammatory lesions reduce PGD2 synthase and increase PGE2 release. ${ }^{33}$ Thus, the relative abundance of specific prostanoids is the result of the expression and activity of specific isomerases, and the predominant expression of PGES over PGDS may lead to increased inflammation and irritation symptoms.

To determine the PG synthesizing enzyme activity in DE, the induction and distribution of COX-1, COX-2, PGES, and PGDS was investigated using an in vivo mouse model. From the quantitative real-time polymerase chain reaction results, COX-2 and PGES mRNA levels were elevated significantly in lacrimal glands and on ocular surfaces (corneas and conjunctivas). However, PGDS mRNA was decreased significantly in the DE condition. Considering the clinical data, which showed elevated PGE2 levels, these result were supportive. Several interesting results from the in vivo study were found. The first is that both lacrimal glands and ocular surfaces induce PGs. Environmental low humidity stress is 
likely to regulate PGs and their synthesizing enzymes in the ocular surface (e.g., corneal and conjunctiva). However, lacrimal gland PG induction also was observed. This phenomenon may be explained by a neural network between the ocular surface and lacrimal gland. Moreover, the upregulation of COX-2 was higher in the lacrimal glands than the ocular surface. Taken together, the lacrimal glands produced more PGE2 than the ocular surface and were the major sources of PGE2 resulting from desiccation stress. Therefore, the neural network between tear-producing tissues may be critical for indirect PG induction. However, a DE model induced by not only desiccating stress, but also injection of scopolamine, was used. It has been reported that pharmacologic cholinergic blockade (scopolamine) stimulates inflammatory cytokine production and lymphocytic infiltration in the mouse lacrimal gland. ${ }^{34}$ So, it is possible that pharmacologic blockade of lacrimal secretion induced changes of PGs in the LG. Future studies will be needed to determine what role the pharmacologic blockade using scopolamine plays in the induction of PG synthesis.

The second interesting result is that COX-2 and PGES did not coexist in the same cells from DE-sensitive tissues. In a previous study, the PGE2 synthesizing key enzymes, COX-2 and PGES, were upregulated by a proinflammatory stimulus and colocalized in the same cells to induce PGE2 effectively, as detected by immunohistochemical staining. ${ }^{35,36}$ However, in the ocular surface and lacrimal glands, both enzymes were expressed in different types of cells (e.g., acinar and ductal epithelial cells vs. periductal infiltrated cells of the lacrimal gland), so PGE2 seemed to be produced by cooperative work between the lacrimal acinar, ductal epithelial, and infiltrated inflammatory cells in the lacrimal gland or cornea and conjunctival epithelium in the ocular surface after desiccation stress. Periductal PG synthesis also was found in infiltrated cells. Because the infiltrated cells expressed PGES, these cells may play a role in PGE2 induction in the lacrimal glands. Considering the topographic expression of COX-2 and PGES in the lacrimal glands, at least some PGE2 precursors (PGH2) may be secreted into the ducts and may convert to PGE2 in periductal infiltrated cells. Finally, PGDS expression was found in the conjunctiva with DE stimulation. However, the cornea expressed only COX-2. Because PGD2 and PGDS have antiinflammatory and protective effects against inflammatory damage, elevated PGDS activity may make the conjunctivalepithelium more resistant to desiccation stress than the cornea.

The role of PG-induced ocular surface nerve firing in DE remains unknown. The elevated firing activity of the corneal nociceptors may stimulate tear production to overcome surface dryness as well as to elicit irritation symptoms. The patient population in this study was divided by DE disease period, and it was found that PGE2 tear levels were higher in patients with recently developed DE (DE diagnosed within 3 months) than in chronic patients (data not shown). Moreover, the COX-2 and PGE2 mRNA levels were more elevated after 3 days than after 7 days in the DE mouse model. Therefore, PGE2 elevation may be responsible for the early compensatory mechanism against ocular surface desiccation to induce more tear production. In addition to lowering the firing threshold and tear secretion, PGE2 also is a potent inducer of inflammation, including inflammatory cell infiltration and angiogenesis. ${ }^{37,38}$ Therefore, elevated PGE2 in DE patients may aggravate ocular surface inflammation by inducing other inflammatory mediators.

Because enhanced excitability of primary sensory neurons in inflammation and pathologic pain states is a major contributor to the perception of pain or irritation, specific pharmacologic agents that specifically dampen aberrant activity are desirable for therapy. ${ }^{3}$ Therefore, COX-2 and PGE2 would be good targets for managing DE. However, the COX-2 levels in the lacrimal glands were not decreased effectively by the topical COX-2-specific inhibitor celecoxib in the in vivo study (Fig 7). This implies that a topical approach alone may be insufficient to reduce ocular surface PGE2 levels effectively. Moreover, the lacrimal gland COX-2 levels are higher than the ocular surface levels and are not well controlled by 
topical drugs. Therefore, more effective ways to suppress lacrimal gland PGE2 induction must be found to suppress its synthesis and reduce patients' irritation symptoms adequately.

The limitation of this study is that tear PG levels were measured with a cross-sectional study design. No studies have been published about PG level in tears in DE, so the concentration of PGs in DE patients was measured and the induction mechanism was investigated using in vivo studies. Use of a specific COX-2 inhibitor in a well-controlled, designed, and masked human study is needed. Also, other substances that may cause ocular irritation, such as substance $\mathrm{P}$ or bradykinin, should be investigated for their relationship to ocular irritation in DE.

In conclusion, this study found that PGE2 levels were elevated significantly in DE patients and correlated well with patient symptoms. Considering the function of PGE2 in nerve receptor firing, this may be one of the main reasons for irritation symptoms and a good therapeutic target. In addition, exploring existing knowledge about pain and noxious substance levels may be beneficiary for DE investigators for interpreting their data and understanding better the pathophysiologic mechanism of irritation symptoms in DE disease.

\section{Acknowledgments}

The authors thanks Hyung Jae Chung for the discussion and interpretation of the immunohistochemical staining data.

Supported by the Basic Science Research Program through the National Research Foundation of Korea (NRF), funded by the Ministry of Education, Science, and Technology, Seoul, Korea (grant no.: 2011-0001384).

\section{References}

1. Johnson ME, Murphy PJ. Changes in the tear film and ocular surface from dry eye syndrome. Prog Retin Eye Res. 2004; 23:449-474. [PubMed: 15219877]

2. Pflugfelder SC, Solomon A, Stern ME. The diagnosis and management of dry eye: a twenty-fiveyear review. Cornea. 2000; 19:644-649. [PubMed: 11009316]

3. Johnson ME. The association between symptoms of discomfort and signs in dry eye. Ocul Surf. 2009; 7:199-211. [PubMed: 19948103]

4. Lemp MA. Advances in understanding and managing dry eye disease. Am J Ophthalmol. 2008; 146:350-356. [PubMed: 18599017]

5. Muller LJ, Marfurt CF, Kruse F, Tervo TM. Corneal nerves: structure, contents and function. Exp Eye Res. 2003; 76:521-542. [PubMed: 12697417]

6. Belmonte C, Gallar J. Cold thermoreceptors, unexpected players in tear production and ocular dryness sensations. Invest Ophthalmol Vis Sci. 2011; 52:3888-3892. [PubMed: 21632706]

7. Acosta MC, Peral A, Luna C, et al. Tear secretion induced by selective stimulation of corneal and conjunctival sensory nerve fibers. Invest Ophthalmol Vis Sci. 2004; 45:2333-2336. [PubMed: 15223813]

8. Belmonte C, Acosta MC, Gallar J. Neural basis of sensation in intact and injured corneas. Exp Eye Res. 2004; 78:513-525. [PubMed: 15106930]

9. Belmonte C. Eye dryness sensations after refractive surgery: impaired tear secretion or "phantom" cornea? J Refract Surg. 2007; 23:598-602. [PubMed: 17598580]

10. Situ P, Simpson TL. Interaction of corneal nociceptive stimulation and lacrimal secretion. Invest Ophthalmol Vis Sci. 2010; 51:5640-5645. [PubMed: 20554608]

11. Dubin AE, Patapoutian A. Nociceptors: the sensors of the pain pathway. J Clin Invest. 2010; 120:3760-3772. [PubMed: 21041958]

12. Weidner C, Schmelz M, Schmidt R, et al. Functional attributes discriminating mechano-insensitive and mechano-responsive C nociceptors in human skin. J Neurosci. 1999; 19:10184-10190.

[PubMed: 10559426] 
13. Tender GC, Walbridge S, Olah Z, et al. Selective ablation of nociceptive neurons for elimination of hyperalgesia and neurogenic inflammation. J Neurosurg. 2005; 102:522-525. [PubMed: 15796388]

14. Namer B, Handwerker HO. Translational nociceptor research as guide to human pain perceptions and pathophysiology. Exp Brain Res. 2009; 196:163-172. [PubMed: 19350229]

15. Huang SM, Lee H, Chung MK, et al. Overexpressed transient receptor potential vanilloid 3 ion channels in skin keratinocytes modulate pain sensitivity via prostaglandin E2. J Neurosci. 2008; 28:13727-13737. [PubMed: 19091963]

16. Bandell M, Story GM, Hwang SW, et al. Noxious cold ion channel TRPA1 is activated by pungent compounds and bradykinin. Neuron. 2004; 41:849-857. [PubMed: 15046718]

17. Meves H. The action of prostaglandins on ion channels. Curr Neuropharmacol. 2006; 4:41-57. [PubMed: 18615137]

18. Alm A. Prostaglandin derivates as ocular hypotensive agents. Prog Retin Eye Res. 1998; 17:291312. [PubMed: 9695796]

19. Hollo G. The side effects of the prostaglandin analogues. Expert Opin Drug Saf. 2007; 6:45-52. [PubMed: 17181451]

20. Malvitte L, Montange T, Vejux A, et al. Measurement of inflammatory cytokines by multicytokine assay in tears of patients with glaucoma topically treated with chronic drugs. Br J Ophthalmol. 2007; 91:29-32. [PubMed: 16943231]

21. Solomon A, Dursun D, Liu Z, et al. Pro- and anti-inflammatory forms of interleukin-1 in the tear fluid and conjunctiva of patients with dry-eye disease. Invest Ophthalmol Vis Sci. 2001; 42:22832292. [PubMed: 11527941]

22. Muraki T, Fujimori K, Ishizaka M, et al. Effects of interleukin-1beta and prostaglandin E2 on prostaglandin D synthase production in cultivated rat leptomeningeal cells. J Cereb Blood Flow Metab. 2004; 24:409-418. [PubMed: 15087710]

23. Tong L, Wong TY. Aspirin and dry eye [letter]. Ophthalmology. 2009; 116:167. [PubMed: 19118708]

24. Lee HK, Ryu IH, Seo KY, et al. Topical $0.1 \%$ prednisolone lowers nerve growth factor expression in keratoconjunctivitis sicca patients. Ophthalmology. 2006; 113:198-205. [PubMed: 16360211]

25. Barabino S, Shen L, Chen L, et al. The controlled-environment chamber: a new mouse model of dry eye. Invest Ophthalmol Vis Sci. 2005; 46:2766-2771. [PubMed: 16043849]

26. Iyer JP, Srivastava PK, Dev R, et al. Prostaglandin E(2) synthase inhibition as a therapeutic target. Expert Opin Ther Targets. 2009; 13:849-865. [PubMed: 19530988]

27. Chen D, Wei Y, Li X, et al. sPLA2-IIa is an inflammatory mediator when the ocular surface is compromised. Exp Eye Res. 2009; 88:880-888. [PubMed: 19116146]

28. Das UN. Essential fatty acids: biochemistry, physiology and pathology. Biotechnol J. 2006; 1:420439. [PubMed: 16892270]

29. Aragona P, Bucolo C, Spinella R, et al. Systemic omega-6 essential fatty acid treatment and PGE1 tear content in Sjogren's syndrome patients. Invest Ophthalmol Vis Sci. 2005; 46:4474-4479. [PubMed: 16303936]

30. Ek M, Engblom D, Saha S, et al. Inflammatory response: pathway across the blood-brain barrier. Nature. 2001; 410:430-431. [PubMed: 11260702]

31. Cipollone F, Fazia M, Iezzi A, et al. Balance between PGD synthase and PGE synthase is a major determinant of atherosclerotic plaque instability in humans. Arterioscler Thromb Vasc Biol. 2004; 24:1259-1265. [PubMed: 15155382]

32. Bazan NG, Allan G. Signal transduction and gene expression in the eye: a contemporary view of the pro-inflammatory, anti-inflammatory and modulatory roles of prostaglandins and other bioactive lipids. Surv Ophthalmol. 1997; 41(suppl):S23-S34. [PubMed: 9154273]

33. Fournier T, Fadok V, Henson PM. Tumor necrosis factoralpha inversely regulates prostaglandin D2 and prostaglandin E2 production in murine macrophages: synergistic action of cyclic AMP on cyclooxygenase-2 expression and prostaglandin E2 synthesis. J Biol Chem. 1997; 272:3106531072. [PubMed: 9388257] 
34. Pitcher JD III, De Paiva CS, Pelegrino FS, et al. Pharmacological cholinergic blockade stimulates inflammatory cytokine production and lymphocytic infiltration in the mouse lacrimal gland. Invest Ophthalmol Vis Sci. 2011; 52:3221-3227. [PubMed: 21273534]

35. Moore AH, Olschowka JA, O'Banion MK. Intraparenchymal administration of interleukin-1beta induces cyclooxygenase-2-mediated expression of membrane- and cytosolic-associated prostaglandin E synthases in mouse brain. J Neuroimmunol. 2004; 148:32-40. [PubMed: 14975584]

36. Ozaki-Okayama Y, Matsumura K, Ibuki T, et al. Burn injury enhances brain prostaglandin E2 production through induction of cyclooxygenase- 2 and microsomal prostaglandin $\mathrm{E}$ syn-thase in cerebral vascular endothelial cells in rats. Crit Care Med. 2004; 32:795-800. [PubMed: 15090964]

37. Liclican EL, Nguyen V, Sullivan AB, Gronert K. Selective activation of the prostaglandin E2 circuit in chronic injuryinduced pathologic angiogenesis. Invest Ophthalmol Vis Sci. 2010; 51:6311-6320. [PubMed: 20610836]

38. Altinoz MA, Korkmaz R. NF-kappaB, macrophage migration inhibitory factor and cyclooxygenase-inhibitions as likely mechanisms behind the acetaminophen- and NSAIDprevention of the ovarian cancer. Neoplasma. 2004; 51:239-247. [PubMed: 15254653] 
A. PGE2

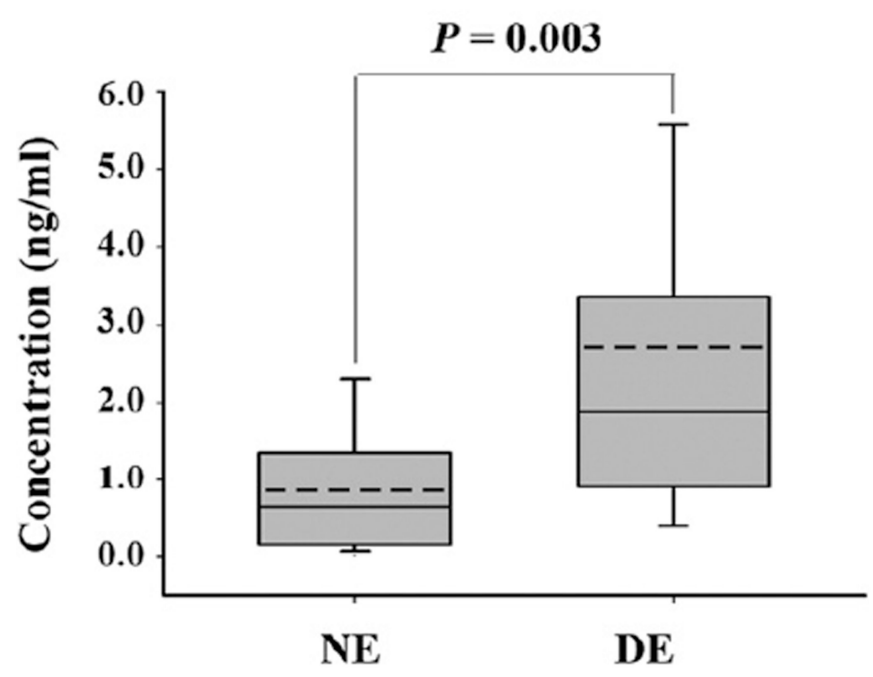

\section{B. PGD2}

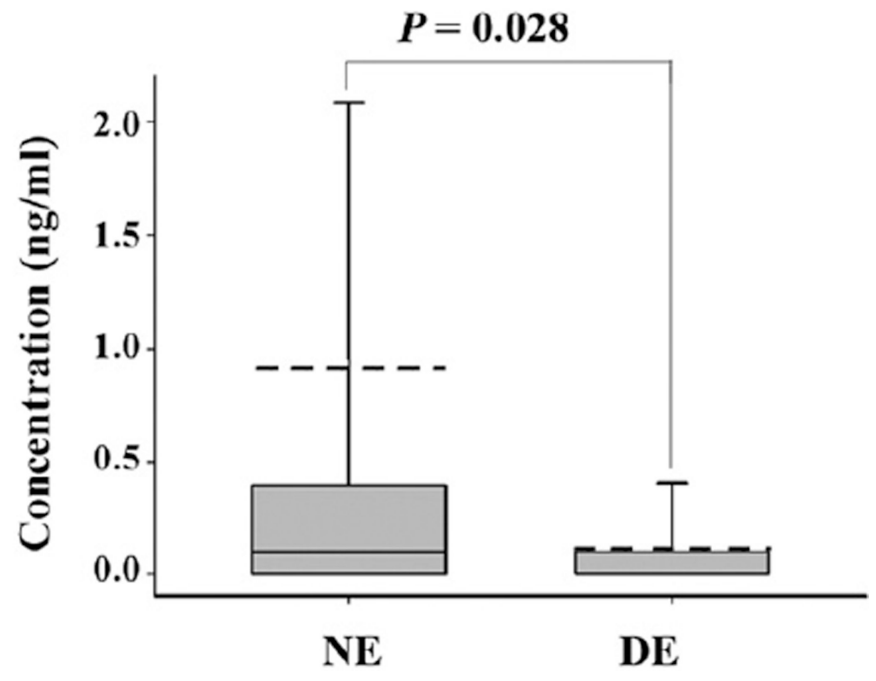

Figure 1.

Graphs showing expression of prostaglandins (PGs) in the tears of controls $(n=33)$ and dry eye patients $(n=46)$. Concentrations of $(\mathbf{A})$ prostaglandin $E(P G E 2)$ and $(\mathbf{B})$ prostaglandin D (PGD2) were measured by nano-liquid chromatography tandem mass spectrometry. The boxplot represents (center bar) the median, (bottom) $25 \%$, and (top) $75 \%$ percentiles. The error bars are the maximum value and the minimum value. The dotted line indicates the mean value. $\mathrm{DE}=$ dry eye; $\mathrm{NE}=$ normal control eye. 
COX-2 mRNA in lacrimal gland

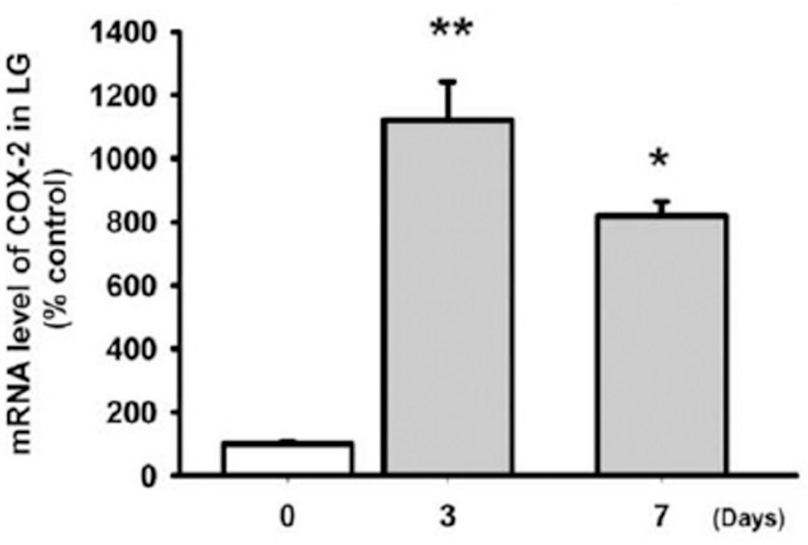

PGES in LG

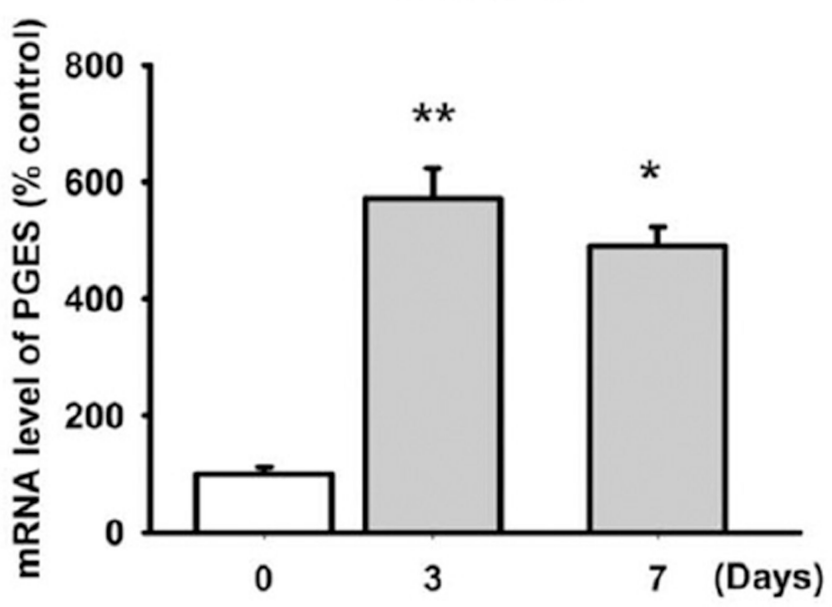

Figure 4. variance). The error bars indicate the standard deviation.

\section{COX-2 mRNA in cornea}

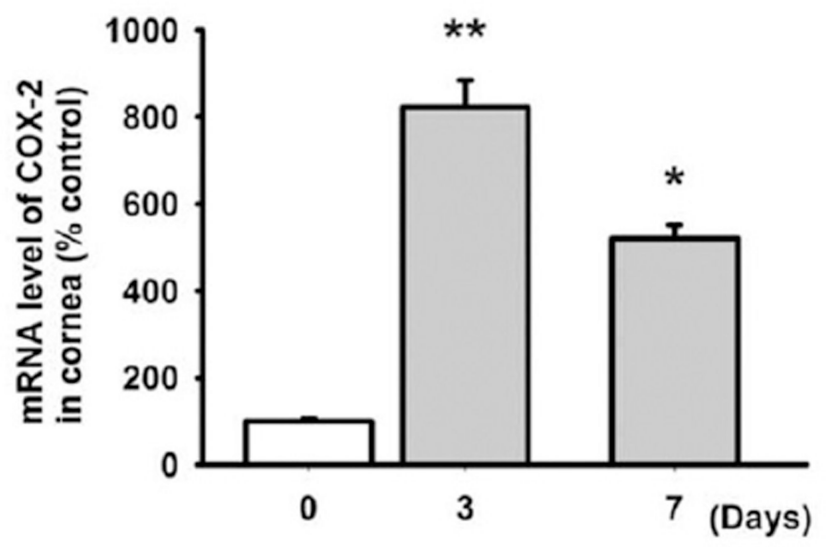

PGDS in LG

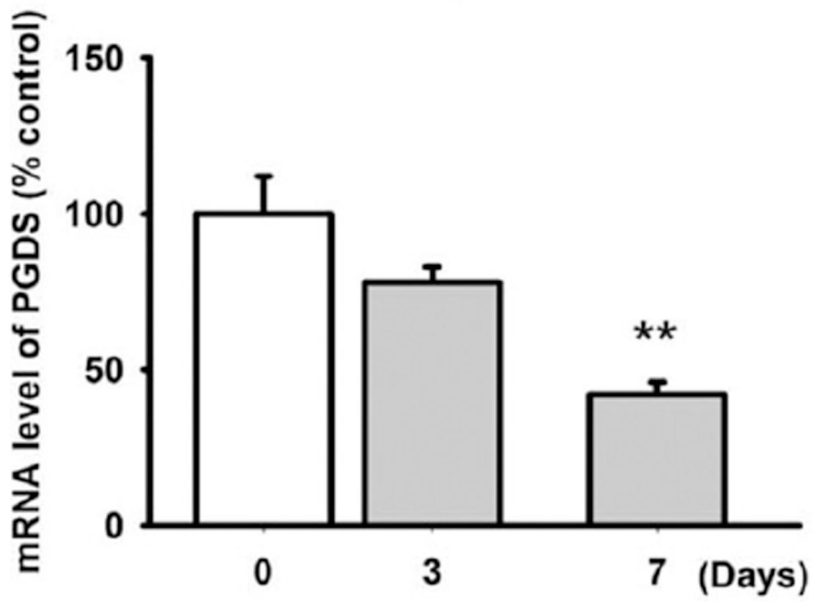

Bar graphs showing quantitation of mRNA expression of cyclooxygenase-2 (COX-2), prostaglandin E synthase (PGES), and prostaglandin D synthase (PGDS). C57B6 female mice $(n=10)$ were housed in a dry eye (DE) chamber for 1 week. Five mice were killed at days 3 and 7 after DE induction in each. mRNA was extracted from lacrimal glands (LGs) and ocular surface (cornea and conjunctiva) after enucleation. Five control mice also were killed. Using quantitative real-time polymerase chain reaction, the levels of COX-2, PGES, and PGDS were measured at each time point $(* P<0.05, * * P<0.01,1$-way analysis of 

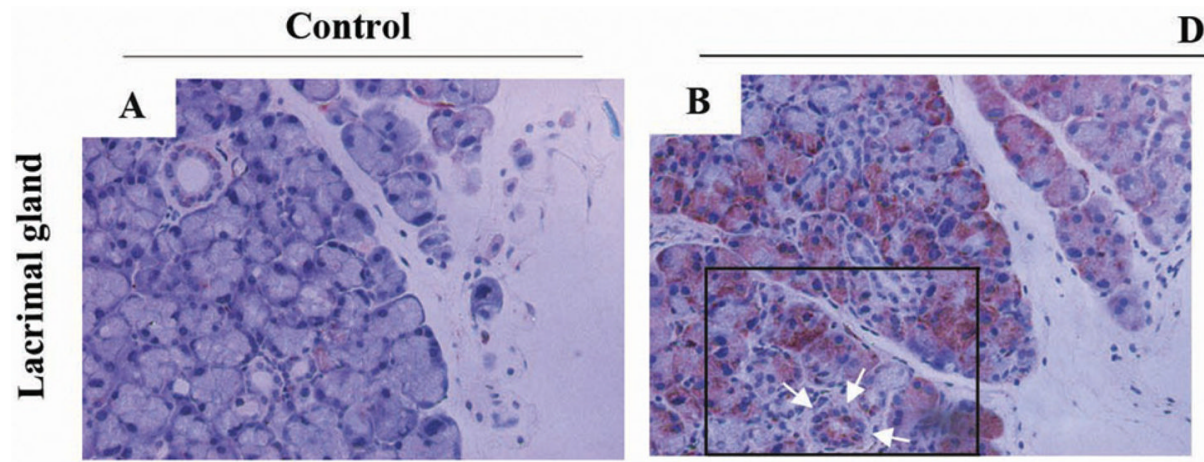

DE
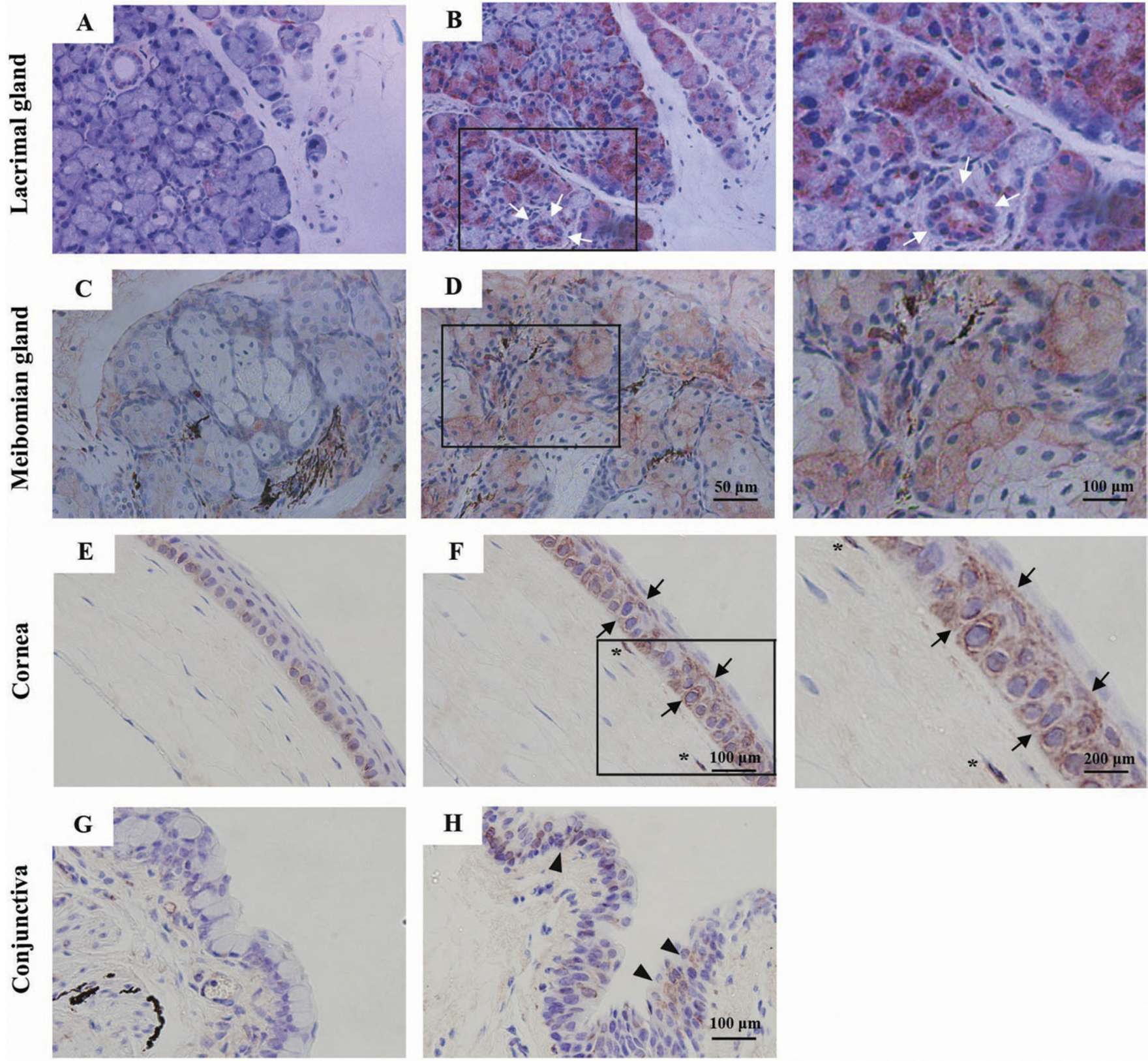

Figure 5.

Photomicrographs showing immunohistochemical staining results of cyclooxygenase-2 $(\mathrm{COX}-2)$ in the ocular surfaces and lacrimal glands. Mouse ocular tissue samples $(\mathrm{n}=5)$ were secured after 7 days in a dry eye chamber, and immunohistochemical staining was performed using antimouse COX-2. Cyclooxygenase-2 expression patterns were determined and compared with $(\mathbf{A}, \mathbf{B})$ healthy control mouse lacrimal glands, $(\mathbf{C}, \mathbf{D})$ meibomian glands, $(\mathbf{E}, \mathbf{F})$ cornea, and $(\mathbf{G}, \mathbf{H})$ conjunctiva. The left column shows the staining pattern from a representative healthy control mouse $(n=5)$. The middle and right columns show the staining pattern from the dry eye mice $(n=5)$. The right column is a magnification of the black box in the middle column. The white arrows (B) indicate ductal epithelial cell 
staining. The black arrows $(\mathbf{F})$ and arrowheads $(\mathbf{H})$ mark the COX-2-positive cells in the cornea and conjunctiva, respectively. 

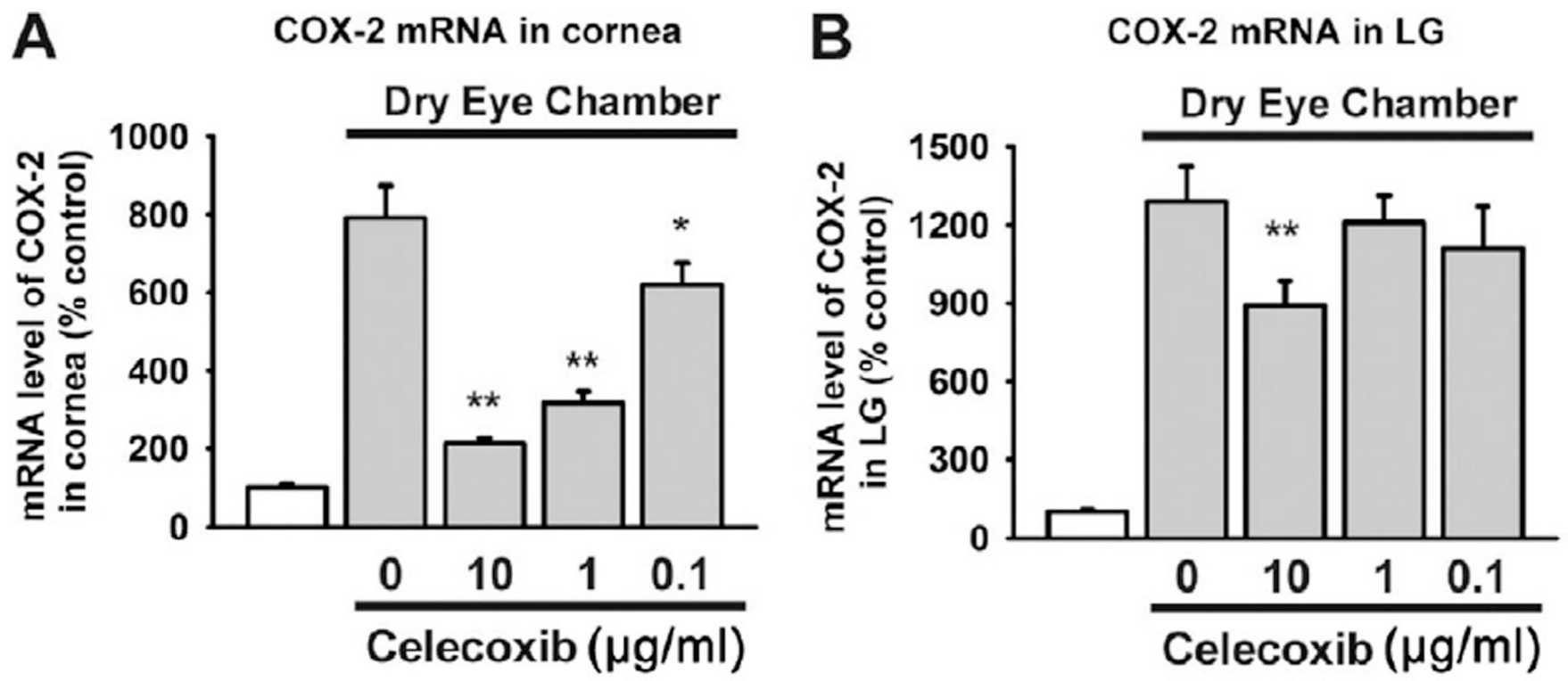

Figure 7.

Bar graphs showing cyclooxygenase-2 (COX-2) mRNA expression in the cornea and lacrimal glands (LGs) after topical celecoxib treatment. C57B6 female mice $(\mathrm{n}=20)$ were housed in a dry eye chamber for 1 week. Then, celecoxib $(0.1 \mu \mathrm{g} / \mathrm{ml}, 1.0 \mu \mathrm{g} / \mathrm{ml}$, and $10.0 \mu \mathrm{g} /$ $\mathrm{ml} 3$ times daily) was instilled in 1 eye for another week. Five mice in each group were killed at 7 days after celecoxib treatment, and the cornea and LGs were removed. Five control mice also were killed. mRNA extraction and quantitative polymerase chain reaction for COX-2 were performed using the same protocols and primers as above $\left({ }^{*} P<0.05\right.$, $* * P<0.01,1$-way analysis of variance). The error bars indicate the standard deviation. 


\section{Table 2}

Correlation of Ocular Irritation Scores and Concentration of Prostaglandin E2 and Prostaglandin D2 in Tears

\begin{tabular}{lrlcc}
\hline & Coefficient & $\boldsymbol{P}$ Value & $\boldsymbol{R}^{\mathbf{2}}$ & $\begin{array}{c}\text { 95\% Confidence } \\
\text { Interval }\end{array}$ \\
\hline PGE2 & 0.17 & 0.16 & 0.03 & -0.07 to 0.41 \\
PGD2 & -0.17 & 0.11 & 0.04 & -0.39 to 0.04 \\
PGE2-to-PGD2 ratio & 3.24 & $0.012^{*}$ & 0.18 & $0.75-5.74$ \\
\hline
\end{tabular}

PGD2 = prostaglandin D2; PGE2 = prostaglandin E2.

* $P<0.05$. 
Table 3

Immunohistochemical Staining of Prostaglandin Synthesizing Enzymes in Ocular Tissues

\begin{tabular}{lccc}
\hline & Cyclooxygenase-2 & Prostaglandin E Synthase & Prostaglandin D Synthase \\
\hline Conjunctiva & $\uparrow$ & $\uparrow$ & $\uparrow$ \\
Epithelium & - & - & - \\
Stroma & $\uparrow$ & - & - \\
Cornea & - & - & - \\
Epithelium & - & - & - \\
Stroma & $\uparrow \uparrow$ & - & - \\
Endothelium & $\uparrow$ & - & - \\
Lacrimal gland & - & $\uparrow$ & - \\
Acina & $\uparrow$ & - & $\downarrow$ \\
Ductal epithelium & - & - & - \\
Periductal infiltrating cell & - & - & \\
Meibomian gland & & & \\
Acina & & & \\
Ductal epithelium & & & \\
\hline
\end{tabular}

$\uparrow=$ elevated expression compared with normal control; $-=$ not detected; $\uparrow \uparrow=$ extensively elevated expression compared with normal control. Especially decreased staining at nucleus. 